\title{
Radiomitigative Effects of Approved Hematopoietic Drugs on Mice Exposed to Lethal Total-body Irradiation
}

\author{
T. Nishida ${ }^{1}$, M. Yamaguchi ${ }^{1}$, S. Miura ${ }^{1}$, K. Waga ${ }^{1}$, N. Kawabata ${ }^{1}$, \\ M. Syaifudin ${ }^{2}$ and I. Kashiwakura ${ }^{1^{\star}}$ \\ ${ }^{I}$ Hirosaki University, Department of Radiation Science, Hirosaki University Graduate School of Health Science, Japan \\ ${ }^{2}$ Center for Radiation Safety Technology and Metrology, National Nuclear Energy Agency, \\ Jl. Lebak Bulus Raya No.49, Jakarta 12440, Indonesia
}

\section{ARTICLE INFO}

Article history:

Received 22 April 2019

Received in revised form 14 October 2019

Accepted 5 December 2019

Keywords:

Approved hematopoietic drugs;

Lethal total-body irradiation;

Acute radiation syndrome;

Radio-mitigator

\begin{abstract}
A B S T R A C T
In cases of radiological accidents, especially for victims exposed to high-dose totalbody irradiation (TBI), the administration of appropriate approved hematopoietic drugs is the most rapid medical treatment for preventing severe acute radiation syndrome, which is associated with a high mortality rate. However, at present, there are few suitable pharmaceutical drugs available in Japan, aside from granulocyte colony-stimulating factor (G-CSF). Depending on the situation surrounding the accident, various drug treatment options and the development of effective drug therapies may be required. In the present study, we assessed various combinations of seven commercially available drugs-G-CSF, erythropoietin (EPO), romiplostim (RP), ancer (AN), cepharanthine (CE), leucon (LC) and leukoprol (LP)-in mice exposed to a lethal dose of 7 or 8 Gy of X-ray irradiation. Each drug was administered as a single or mixed intraperitoneal injection once or twice daily for three consecutive days. The single administration of the approved hematopoietic drugs CE, LC, or LP twice a day for 3 days significantly improved the 30-day survival rate of lethal TBI mice ( $p<0.05 ; 75 \%, 100 \%$, or $100 \%$, respectively) compared with the untreated TBI mice, accompanied by a gradual increase in the body weight. Furthermore, the combined administration of RP, EPO and G-CSF or single administration of RP alone gradually increased the body weight of mice exposed to lethal TBI, with 30 -day survival rates of $75 \%$ or $100 \%$, respectively $(p<0.05)$. This study suggested that some new domestically approved hematopoietic drugs may have radiomitigative potential for mice exposed to lethal TBI, and the 12-h interval administration of LC or LP for 3 days to 7-Gy-TBI mice and 12-h interval administration of RP alone for 3 days to 8-Gy-TBI mice were the most suitable medications with respect to the 30-day survival rate. As long as the threat of nuclear disaster exists, diverse efforts in the field of radiation emergency medicine, including the development of effective drug therapies, will be necessary.
\end{abstract}

\section{INTRODUCTION}

In the event of an unexpected radiation accident, high-dose exposure exceeding the lethal dose (>2 Gy) can lead to death due to acute radiation syndrome (ARS), which is caused by myelosuppression and intestinal disorder [1,2]. Medication is a promptly applicable initial treatment

*Corresponding author.

E-mail address: h18gg208@hirosaki-u.ac.jp

DOI: https://doi.org/10.17146/aij.2020.950 when multiple victims are exposed, but at present, there are few suitable pharmaceutical drugs available. The International Atomic Energy Agency (IAEA) recommends medication with the combined administration of hematopoietic cytokines, including granulocyte colony-stimulating factor (G-CSF), granulocyte macrophage-colony stimulating factor (GM-CSF), interleukin-3 (IL-3) and thrombopoietin (TPO), to accelerate the bone marrow (BM) recovery of victims exposed to lethal doses of totalbody irradiation (TBI) [3]. The human recombinant 
hematopoietic cytokine G-CSF is used clinically worldwide [4,5], while human recombinant GM-CSF, IL-3 and TPO are used in a few countries (but not in Japan). Therefore, the establishment of an optimum therapeutic protocol using only currently approved pharmaceutical drugs to rescue victims exposed to lethal TBI in case of emergency is desired. Although substantial efforts have been put into developing radioprotective/ radiomitigative agents, little success has been achieved. To resolve this situation, we have been developing radioprotective/radiomitigative agents using several approved pharmaceutical drugs. Our recent report showed that, among the currently approved pharmaceutical drugs, romiplostim (RP) is the most suitable candidate for victims exposed to high-dose TBI [6,7]. However, depending on the situation of the accident, various drug treatment options may be required.

In the present study, to establish an optimum therapeutic protocol using currently approved hematopoietic drugs to increase the survival of victims exposed to lethal TBI, we assessed various combinations of seven commercially available drugs in mice exposed to a lethal dose of X-ray irradiation: G-CSF, erythropoietin (EPO), RP, ancer (AN), cepharanthine (CE), leucon (LC) and leukoprol (LP).

\section{EXPERIMENTAL METHODS}

\section{Lethal x-ray TBI in mice}

Seven-week-old female C57BL/6J Jcl mice were delivered from the breeding facilities of Clea Japan (Tokyo, Japan) and housed in a conventional animal room with a 12-h light/dark cycle. These mice had ad libitum access to sterilized standard laboratory mouse chow diet and drinking water. At 8 weeks of age, the mice were subjected to lethal TBI at 7 or 8 Gy of X-rays $(150 \mathrm{kVp}, 20 \mathrm{~mA}$, $0.5 \mathrm{~mm}$ aluminum and $0.3 \mathrm{~mm}$ copper filters) at a dose rate of $1.0 \mathrm{~Gy} / \mathrm{min}$ using an X-ray generator (MBR-1520R; Hitachi Medical Co., Tokyo, Japan). The mice were then administered with the medications as described below. The sizes of each experimental group of mice are indicated in the figure legends. All experiments were conducted according to the legal regulations and the Guidelines for Animal Experiments of Hirosaki University after approval by the animal experimental committees. In the present study, the selection criteria applied prior to sacrifice were $>20 \%$ loss of body weight and respiratory distress.

\section{Treatment regimen using combinations of seven hematopoietic drugs}

The irradiated mice were administered with different types of medication within 2- $\mathrm{h}$ after TBI, and mice without any treatment were used as controls. The combinations of medications were composed of the following commercially available drugs in Japan: recombinant human G-CSF, Neutrogena ${ }^{\circledR}$ (Chugai Pharmaceutical Co., Ltd., Tokyo, Japan); recombinant human EPO, Espo ${ }^{\circledR}$ (Kyowa Hakko Kirin Co., Ltd., Tokyo, Japan); human thrombopoietin receptor agonist RP, Romiplate $^{\circledR}$ (Kyowa Hakko Kirin Co., Ltd.); AN,

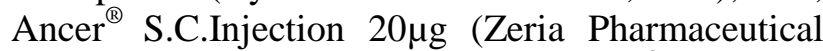
Co., Ltd., Tokyo,Japan); LC, Leucon ${ }^{\circledR}$ Injection (Ohara Pharmaceutical Co., Ltd., Tokyo, Japan); CE, Cepharanthin ${ }^{\circledR}$ (Kaken Shoyaku Co., Ltd., Tokyo, Japan); and LP, Leukoprol ${ }^{\circledR}$ (JCR Pharmaceuticals Co., Ltd., Hyogo, Japan). Each drug was administered as a single or mixed in intraperitoneal injection (i.p.) once or twice daily for 3 consecutive days. The dose of G-CSF, EPO, RP, $\mathrm{AN}, \mathrm{LC}, \mathrm{CE}$, and LP were $300 \mu \mathrm{g} / \mathrm{kg}$ of body weight/day, $100 \mathrm{U} / \mathrm{kg}$ of body weight $/$ day, $50 \mu \mathrm{g} / \mathrm{kg}$ of body weight/day, $20 \mu \mathrm{g} / \mathrm{ml}, \quad 5.7 \mathrm{mg} / \mathrm{ml}$, $5.0 \mathrm{mg} / \mathrm{ml}$, and $2.0 \times 10^{5} \mathrm{U} / \mathrm{ml}$, respectively. The treated mice were kept until day 30 , and weighed every week.

\section{Statistical analyses}

Data from survival studies data were analyzed using the Kaplan-Meier method followed by the Mantel-Cox (log-rank) test for the assessment of significant differences. The levels of significance were calculated using Excel 2007 (Microsoft, Redmond, WA, USA) with Statcel 3 add-in software (OMS, Saitama, Japan). A $p$ value of less than 0.05 was taken to indicate statistical significance.

\section{RESULTS AND DISCUSSION}

\section{Administration of drugs improves the 30-day survival of lethal TBI mice}

In order to evaluate the potential in vivo effects of domestically approved hematopoietic drugs on the survival of mice exposed to a lethal 7-Gy dose of X-ray irradiation, four drugs (AN, LC, $\mathrm{CE}$ or LP) were administered to mice once per day for 3 consecutive days within 2-h after TBI. The animals' body weight and survival rate over a period of 30 days were evaluated. The non-irradiated mice that received each drug showed no sign of stress or body weight loss, and zero mortality 
(data not shown). The lethal dose used in the present study was determined based on the 30-day survival noted in various pilot experiments, ranging from 6 to $10 \mathrm{~Gy}$, and we decided to expose the mice to a 7-Gy dose of X-ray irradiation, which resulted in a 30-day survival rate of $25 \%$, as shown in Fig. 1.

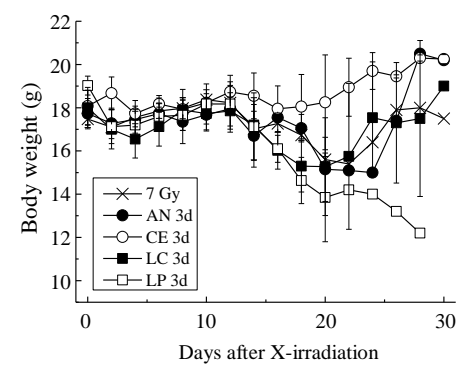

(a)

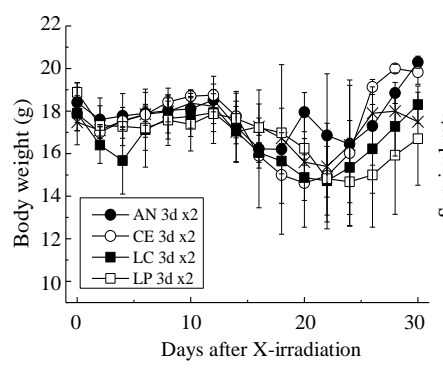

(c)

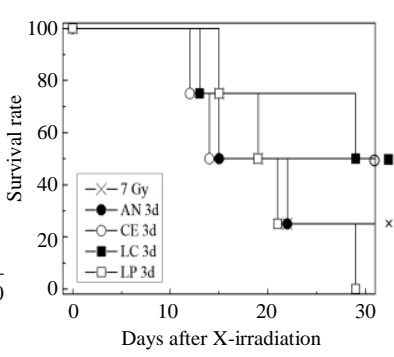

(b)

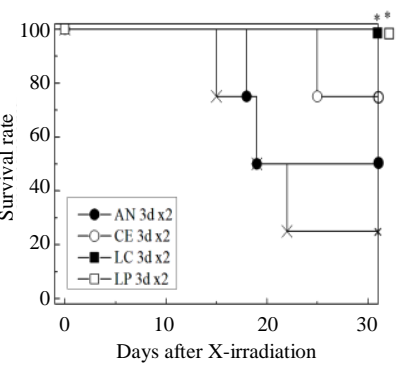

(d)
Fig. 1. Body weight changes and Kaplan-Meier plots of the survival of female C57BL6/J mice. [A] Mice were exposed to 7 Gy of X-rays and administered daily doses of AN, CE, LC or LP for 3 consecutive days via i.p. injection. The first dose was administered within $2 \mathrm{~h}$ post-TBI, and control mice were irradiated and received injections of vehicle. Body weight changes in the surviving mice $(n=4$ per each group) and $[B]$ Kaplan-Meier plots of the survival of mice treated with each drug ( $\mathrm{n}=4$ per each group) are shown. [C] Mice were exposed to $7 \mathrm{~Gy}$ of X-rays and received AN, CE, LC or LP twice per day (12-h interval) for 3 consecutive days via i.p. injection. Body weight changes in the surviving mice $(n=4$ per each group) and [D] Kaplan-Meier plots of the survival of mice treated with each drug ( $\mathrm{n}=4$ per each group) are shown. $* p<0.05$ vs. TBI with vehicle

The body weights of the irradiated mice that received $\mathrm{CE}$ were found to gradually increase with time over a period of 30 days (Fig. 1(a)). The weight of the surviving mice treated with AN or LC gradually decreased and recovered 20 days after TBI. The final 30-day survival rates of mice that received $\mathrm{AN}, \mathrm{CE}$, and $\mathrm{LC}$ were $25 \%, 50 \%$ and $50 \%$, respectively (Fig. 1(b)), which were not significantly improved compared to the mice receiving TBI only. LP had no radiomitigative effects, and treated mice survived until day 28 with accompanying body weight loss. We next tried to overcome the mortality of mice due to 7-Gy TBI by administering each drug twice per day (12-h intervals) for 3 consecutive days. The body weights of irradiated mice that received AN, LC, CE or LP decreased until day 20 and then gradually increased, a trend similar to that in the TBI group (Fig. 1(c)). LC or LP administration twice daily for 3 consecutive days, beginning within 2-h after TBI, at $5.7 \mathrm{mg} / \mathrm{ml}$ or $2.0 \times 10^{5} \mathrm{U} / \mathrm{ml}$, respectively, was able to completely suppress the lethal effects of $7 \mathrm{~Gy}$ of TBI $(p<0.05$; Fig. 1(d)). Furthermore, a $75 \%$ survival rate was observed at day 30 post-TBI when $\mathrm{CE}$ was administered twice daily for 3 consecutive days at $5.0 \mathrm{mg} / \mathrm{ml}$. However, the treatment of mice exposed to lethal TBI with AN resulted in a 30-day survival rate of only $50 \%$, and there was no marked improvement in the survival rate by increasing the frequency of administration.

Based on the above results, the 12-h interval administration of LC, LP or CE was deemed the most suitable in terms of the radiomitigative effects for ARS in individuals exposed to lethal 7-Gy X-ray irradiation. The administration twice per day is more effective to the mice exposed to TBI than once daily administration. CE, LC and LP, which are especially shown the almost or complete rescue of mice exposed to lethal $\mathrm{X}$-irradiation, are all clinically applicable as therapeutic agents for leukopenia in Japan.

$\mathrm{CE}$ is reportedly effective against radiotherapy-induced leukopenia and can show exert anti-inflammatory action by suppressing the activation of nuclear factor-kappa B [8-10]. LC is taken up by BM cells, used for the nucleic acid synthesis to promote the production of leukocytes, and exerts anti-inflammatory action by activating adenylyl cyclase/cyclic adenosine monophosphatedependent protein kinase A [11-13]. LP induces the production of GM-CSF, G-CSF and IL-8; enhances the function of granulocytes; and increases the number of peripheral mononuclear cells in animal models [14]. However, AN, which showed a 30-day survival rate of only $50 \%$ in lethally irradiated mice, induces various types of immune activity, such as protective effects against bacterial and viral infections [15-18], and inhibition of tumor growth [19]. All of the above hematopoietic drugs have the potential to increase blood cell production and suppress the inflammatory response, which may lead to the improvement of the 30-day survival in mice exposed to TBI.

\section{Combined administration of drugs improves the 30-day survival of lethally irradiated mice}

As doses exceed 8-10 Gy, full-blown radiation-induced gastrointestinal syndrome with diarrhea, weight loss, dehydration, sepsis and intestinal bleeding ensues, eventually leading to mortality. We, therefore exposed some mice to higher doses of single-fraction TBI (8 Gy). At this radiation dose, the entire cohort of mice died before 
15-days post-TBI, accompanied by weight loss (Fig. 2(a)). No marked increase in the survival time of TBI mice was observed with the administration of LC or LP twice per day for 3 consecutive days, even though this regimen completely suppressed the lethal effects of 7-Gy irradiation (Fig. 2(b)). In our previous study involving mice lethally irradiated with 7 Gy of $\gamma$-rays, we successfully optimized a protocol with multiple approved drugs: RP, EPO and G-CSF.

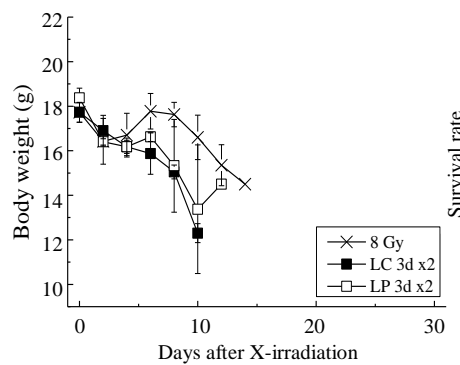

(a)

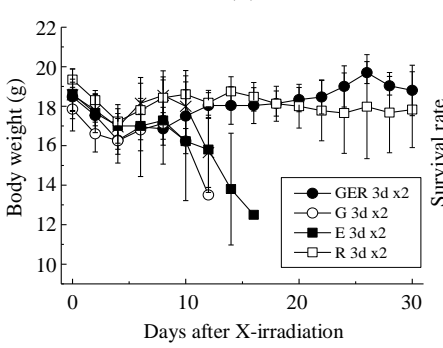

(c)

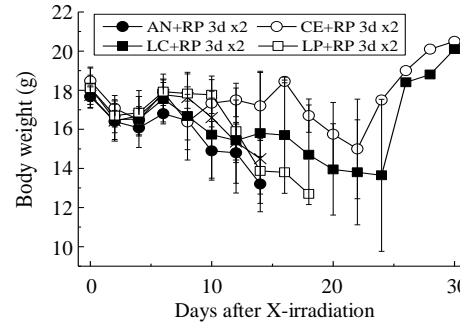

(e)

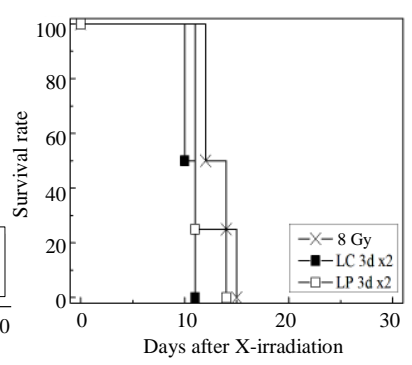

(b)

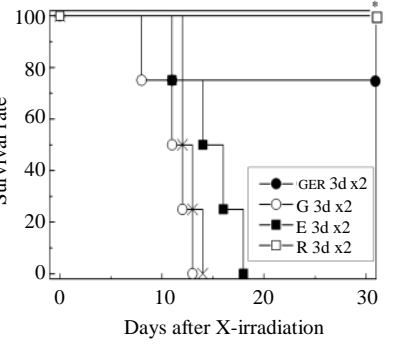

(d)

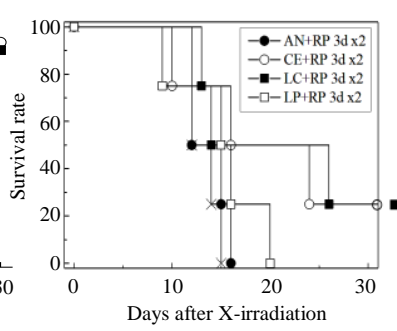

(f)
Fig. 2. Body weight changes and Kaplan-Meier plots of the survival of female C57BL6/J mice. [A] Mice were exposed to 8 Gy of X-rays and received LC or LP twice per day (12-h interval) for 3 consecutive days via i.p. injection. The first dose was administered within $2 \mathrm{~h}$ post-TBI, and control mice were irradiated and received injections of vehicle. Body weight changes in the surviving mice ( $\mathrm{n}=4$ per each group) and [B] Kaplan-Meier plots of the survival of mice treated with each drug ( $\mathrm{n}=4$ per each group) are shown. [C] Mice were exposed to 8 Gy of X-rays and received G-CSF (G), EPO (E), RP (R) or the combination of the 3 drugs (GER) twice per day (12-h interval) for 3 consecutive days via i.p. injection. Body weight changes in the surviving mice $(n=4$ per each group) and [D] Kaplan-Meier plots of the survival of mice treated with each drug $(n=4$ per each group) are shown. $* p<0.05$ vs. TBI with vehicle. [E] Mice were exposed to $8 \mathrm{~Gy}$ of X-rays and received AN, CE, LC or LP each in combination with RP twice per day (12-h interval) for 3 consecutive days via i.p. injection. Body weight changes in the surviving mice $(\mathrm{n}=4$ per each group) and [F] Kaplan-Meier plots of the survival of mice treated with each drug ( $n=4$ per each group) are shown.
The 30-day survival rate of mice treated with these drugs in combination improved to $100 \%$ [20]. We therefore evaluated the radio-mitigative effects of the combination treatment or treatment with each hematopoietic drug twice daily for 3 consecutive days in mice exposed to lethal 8-Gy X-ray irradiation. The combined administration of RP, EPO and G-CSF or single administration of RP alone gradually increased the body weight of mice exposed to lethal TBI, with 30-day survival rates of $75 \%$ or $100 \%$, respectively ( $p<0.05$; Fig. 2(c), (d)). The average post-TBI survival time of mice receiving G-CSF or EPO administration was day 13 or 18 , respectively, and not longer than that of untreated TBI mice. This result suggested that mice receiving $50 \mu \mathrm{g} / \mathrm{kg}$ of $\mathrm{RP}$ twice per day (12-h intervals) for 3 consecutive days would achieve a 30-day survival rate of $100 \%$ after lethal TBI. To establish optimum therapeutic options using currently approved hematopoietic drugs with the goal of increasing the survival rate of victims exposed to lethal TBI following accidental exposure, we assessed the administration of AN, CE, LC, or LP in combination with RP twice per day for 3 consecutive days (Fig. 2(e), (f)). As shown in Fig. 2(f), the combination of CE and RP as well as that of LC and RP resulted in a $25 \%$ survival of irradiated individuals on day 30 . Other combinations (AN and RP or LP and RP) had no radiomitigative effects, but mice that received the combination of LP and RP survived until day 20 (Fig. 2(f)). Based on these results, the 12-h interval administration of RP alone for 3 days to 8-Gy-TBI mice was the most suitable medication with respect to the 30-day survival rate.

G-CSF and EPO are clinically applied worldwide to accelerate the recovery of damaged BM [21-24]. The receptors of G-CSF, EPO and RP are constitutively expressed on the surface of hematopoietic stem cells and these lineages are associated with the differentiation to granulocytes, erythrocytes and megakaryocytes, respectively [25-27]. Each cytokine acts synergistically to exert hematopoietic activity [25-27], induces the activation of the Janus kinase/signal transducers and activators of transcription pathway, and plays an important role in various cell functions, such as the cell survival, proliferation, hematopoiesis and immune response [28]. Therefore, the administration of certain domestically approved drugs is expected to promote the suppression of various stresses or encourage the production/proliferation of hematopoietic cells. However, RP alone was found to effectively improve the condition of mice exposed to a lethal 8-Gy dose of X-ray irradiation (Fig. 2(d)). $\mathrm{RP}$ reportedly causes a rapid increase in the 
mesenchymal stromal/stem cells and common myeloid-erythroid progenitors in the spleen, helping to regulate the function of stem cells by maintaining the microenvironmental stem cell niche instead of the damaged BM [6,7]. In addition, megakaryocyte haematopoiesis in the spleen and lung was shown to be promoted by RP treatment, resulting in the suppression of thrombocytopenia induced by ARS. The present findings showed that the combination of G-CSF, EPO and RP or the single administration of RP alone may be a useful countermeasure for victims accidentally exposed to lethal irradiation. However, the detailed mechanisms underlying the increased survival rate need to be clarified. Additional investigations are currently underway to clarify how the treated mice were able to escape radiation-induced death after TBI, which is usually accompanied by death due to $\mathrm{BM}$ and gastrointestinal dysfunction. Highly effective and completely safe medical countermeasures are needed in order to provide better ways to prevent and control unexpected radiation accidents in the future.

\section{CONCLUSION}

The present findings indicate that certain domestically approved hematopoietic drugs have some radiomitigative potential for mice exposed to lethal TBI According to our results, the 12-h interval administration of LC or LP for 3 days to 7-Gy-TBI mice and the 12-h interval administration of RP alone for 3 days to 8 -Gy-TBI mice were the most suitable regimens with respect to the 30-day survival rate. However, many issues remain to be addressed, such as the safety and efficacy of these regimens in humans, the optimal doses of the drugs, the optimal duration and timing of administration and the applicable range of radiation doses that can be effectively countered. As long as the threat of nuclear disaster exists, diverse efforts in the field of radiation emergency medicine, including the development of effective drug therapies, will be necessary.

\section{ACKNOWLEDGMENT}

This work was supported by a KAKENHI Grant-in-Aid for Scientific Research (A) (No. 16H02667 IK).

\section{REFERENCES}

1. V.K. Singh, P.L. Romaine, V.L. Newman et al., Expert Opinion on Therapeutic Patents 26 (2016) 1399.

2. S.S. Acharya, W. Fendler, J. Watson et al.,
Science Translational Medicine 7 (2015) 287ra69.

3. Anonymous, Diagnosis and Treatment of Radiation Injuries, Safety Report Series 2, International Atomic Energy Agency (1998).

4. V.K. Singh, V.L. Newman and T.M. Seed, Cytokine 71 (2015) 22.

5. H.M. Mehta, M Malandra and S.J. Corey, J. Immunol. 195 (2015) 1341.

6. M. Yamaguchi, T. Hirouchi, K. Yokoyama et al., Scientific Reports 8 (2018) 10659.

7. M. Yamaguchi, T. Hirouchi, H. Yoshioka et al., Free Radic. Biol. Med. 136 (2019) 60.

8. Y.A. Samura, H.S. Said, N.M. Elsherbiny et al., Life Sciences 157 (2016) 187.

9. B. Fernández-Gil, A.E.A. Moneim, F. Ortiz et al., PLoS One 12 (2017) e0174474.

10. K. Manna, U. Das, D. Das et al., Free Radical Research 49 (2015) 422.

11. Y.F. Cheng, G.H. Young, T.M. Chiu et al., Exp. Dermatol. 25 (2016) 162.

12. T. Tomita, H. Goto, K. Sumiya et al., Clinical Psychopharmacology and Neuroscience $\mathbf{1 4}$ (2016) 391 .

13. T. Fukuda, K. Majumder, H. Zhang et al., J. Agric. Food Chem. 64 (2016) 4227.

14. T. Hidaka, S. Akada and A. Teranishi, Cancer Science 94 (2003) 814.

15. K. Katsunuma, K. Yoshinaga, Y. Ohira et al., Molecular immunology 64 (2015) 218.

16. T. Horii, K. Yoshinaga, N. Kobayashi et al., Biol. Pharm. Bull. 37 (2014) 642.

17. M. Kobayashi, D.N. Herndon, R.B. Pollard et al., Immunol. Lett. 40 (1994) 199.

18. H. Sasaki, M. Kobayashi, Y. Emori et al., Biotherapy 10 (1997) 139.

19. T. Sugiyama, K. Fujiwara, Y. Ohashi et al., Annals of Oncology 25 (2014) 1011.

20. T. Hirouchi, K. Ito, M. Nakano et al., Curr. Pharm. Biotechnol. 17 (2015) 190.

21. V.K. Singh, P.L. Romaine and T.M. Seed, Health Phys. 108 (2015) 607.

22. W. Qi, Q. Shen, L. Zhang et al., Exp. Ther. Med. 11 (2016) 2221.

23. I.V. Gilevich, T.V. Fedorenko, I.A. Pashkova et al., Bull. Exp. Biol. Med. 162 (2017) 684. 
24. S.J. Chan, C. Love, M. Spector et al., Neurochem. Int. 107 (2017) 57.

25. S.Q. Zhao and J.M. Li, Zhonggouo Shi Yan Xue Ye Xue Za Zhi. 23 (2015) 871.

26. U. Gutti, S.R. Pasupuleti, I. Sahu et al., Crit.
Rev. Oncol. Hematol. 108 (2016) 175.

27. L.N. Varghese, J.P. Defour, C. Pecquet et al., Front Endocrinol. 8 (2017) 59.

28. R. Morris, N.J. Kershaw, J.J. Babon, Protein Sci. 27 (2018) 1984. 